\title{
Alternating,Recurrent Tolosa Hunt Syndrome -A Rare Case Report
}

\author{
Lekhra OP ${ }^{1}$, Patel $\mathrm{K}^{2}$, Maheshwari $\mathrm{A}^{1}$, Rathore $\mathrm{Y}^{1}$ \\ Department of Neurology ${ }^{l}$ and Radiology ${ }^{2}$ \\ Sri Aurobindo Medical College and PG Institute,Indore India
}

\begin{abstract}
Tolosa Hunt Syndrome(THS) is acute ophthalmoplegia with or without involvement of optic nerve and ophthalmic division of trigeminal nerve and responding promptly to steroid treatment. Its recurrence is well known on the same side but involvement of the alternating side is quite rare. We describe 23 yr female patient who had MRI documented involvement of alternate eye separately at an interval of two years and the symptoms had complete resolution with steroids at both occasions. MRI documented THS has been rarely reported.
\end{abstract}

Key Words : Tolosa-Hunt Syndrome, Opthalmoplegia , Alternating ,Recurrence, MRI

\section{Introduction}

Painful acute ophthalmoplegia with or without involvement of optic nerve and ophthalmic division of trigeminal nerve and responding promptly to steroid treatment has come to be regarded as a distinct clinical entity ,often called Tolosa-Hunt syndrome (THS). The lesions may be in the the cavernous sinus or superior orbital fissure (Gladstone,2007).The recurring nature of THS on the ipsilateral side has been known since its first description. But impairment of the contralateral cavernous sinus/superior orbital fissure, known as alternating THS, is very rare. We report a unusual case of alternating THS wherein the patient has painful opthalmoplegia in alternate eyes at an interval of two years.

\section{Case Report}

A $25 \mathrm{yr}$ old woman presented with retroorbital pain of ten days duration. It was left sided continuous ,nonthrobbing, moderately severe in intensity and associated with vomiting.It had with no diurnal or postural variation.Diplopia was present in all directions of gaze, it disappeared on closure of left eye. There was no history of visual loss,,trauma,dysphagia or change in voice. Examination revealed dilated pupil with impaired eye movements suggestive of left complete third nerve palsy.Right eye examination was normal. Fundus both sides normal .Other cranial nerves and neurological examination was unremarkable . Routine blood counts and blood biochemistry were normal. P-ANCA ,C-ANCA,and APLA were all negative.CSF examination was unremarkable. Visual evoked responses suggested mild delay of latency and decreased amplitude of P100 wave on left side suggesting left anterior pathway dysfuntion but had normal parameters on right side.MRI reveals ill defined isointense soft tissue thickening, along the lateral wall of left cavernous sinus extending into left optic canal (Fig1a) . MRI post contrast axial shows intense enhancement of the lesion with minimal compression over the optic nerve(Fig 1b) Patient was put on oral methyl prednisalone $16 \mathrm{mg}$ twice daily for 5 days then 16 mg once daily for 15 days. Pain resolved in few days and the eye movements improved over two weeks.

Two years back she was admitted with similar complaints of the right eye. In the last admission she had more florid symptoms and signs as she had presented after 25 days. Examination of her right eye had revealed pallor in fundus and the vision was almost nil .Ocular movements were absent in all directions suggesting right third ,fourth and sixth nerve palsy.Left eye was normal Rest of the neurological examination was unremarkable. Routine blood counts and biochemistry were unremarkable. Visual evoked responces showed absent P100 wave on right side indicating gross anterior visual pathway dysfunction. Left P100 wave had normal parameters.MRI revealed the ill defined isointense soft tissue thickening along the lateral wall of right cavernous sinus with extension in to the optic canal(Fig2a).After contrast the same lesion showed diffuse intense enhancement with minimal compression over the right optic nerve (Fig2b). Oral administration of methyprednisolone $16 \mathrm{mg}$ twice daily was initiated and continued for a month and then on taperral doses.. All symptoms resolved and ophthalmoplegia improved dramatically following this treatment. Neurological examination was normal after two months and then patient was lost to follow up. 


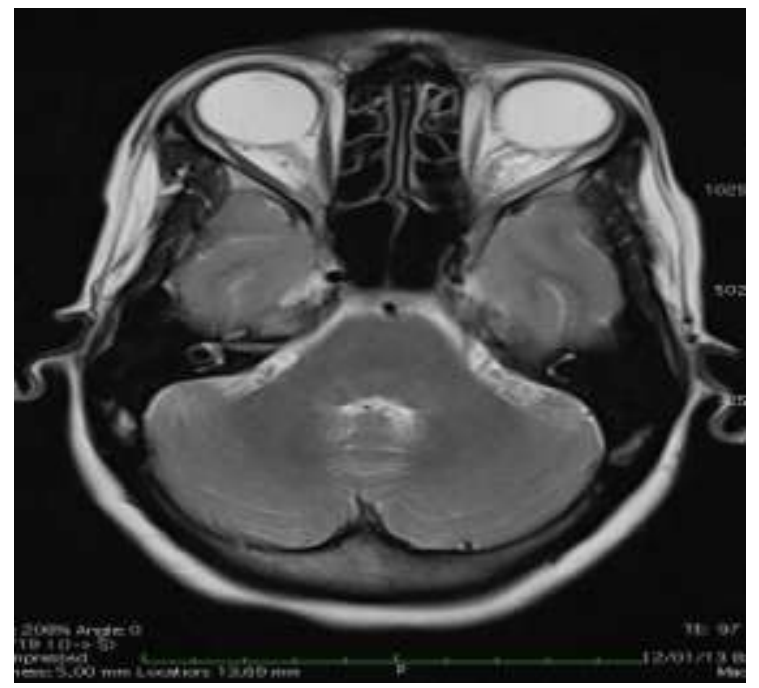

Fig 1a .MRI T2 axial shows ill-defined isointense soft tissue thickening along the lateral wall of left cavernous sinus extending into optic canal .

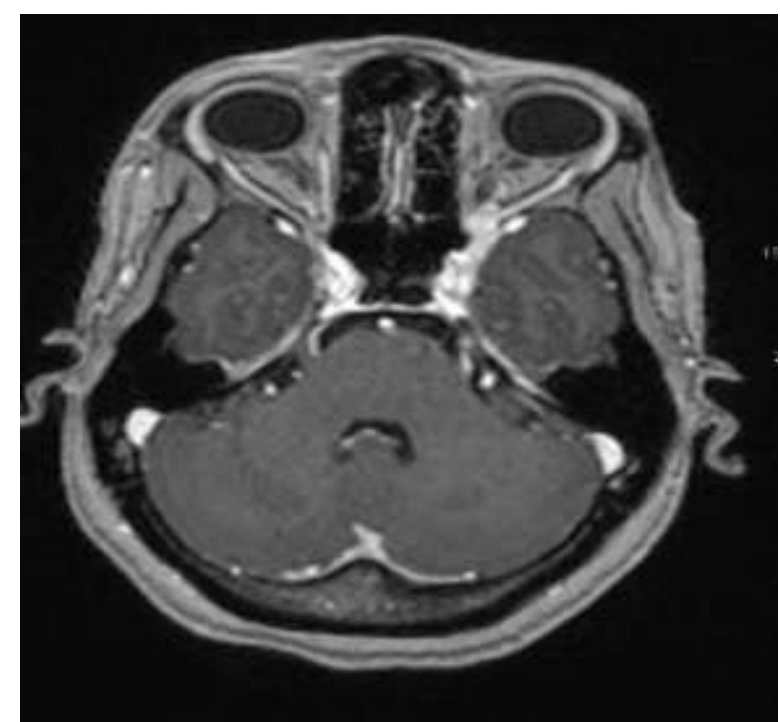

Fig. $1 \mathrm{~b}$ post contrast shows intense enhancement of the same lesion on the left side with mild compression over the optic nerve.

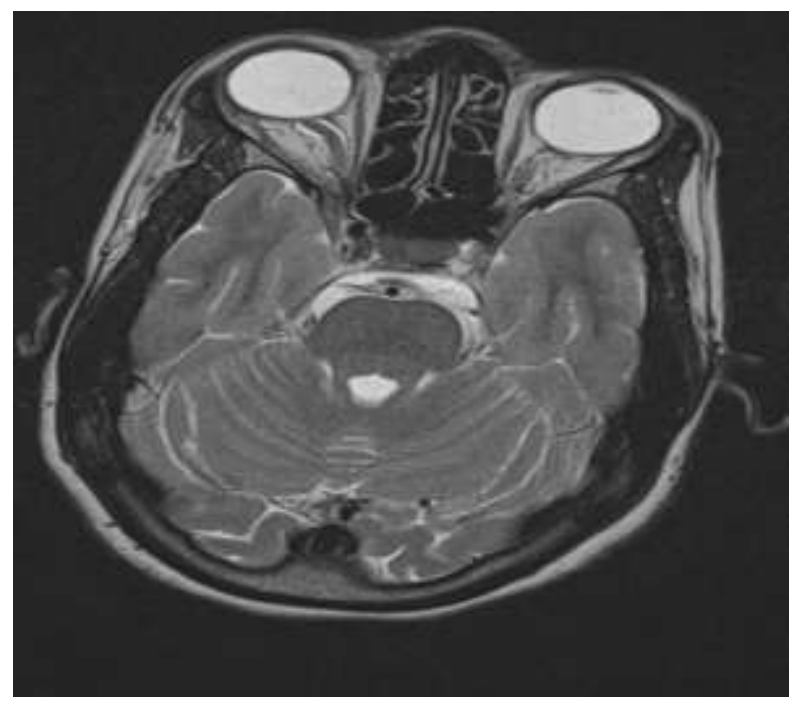


Fig 2a MRI T2 axial illdefined isointense soft tissue thickening along the lateral wall of right cavernous sinus with extension in to the optic canal.

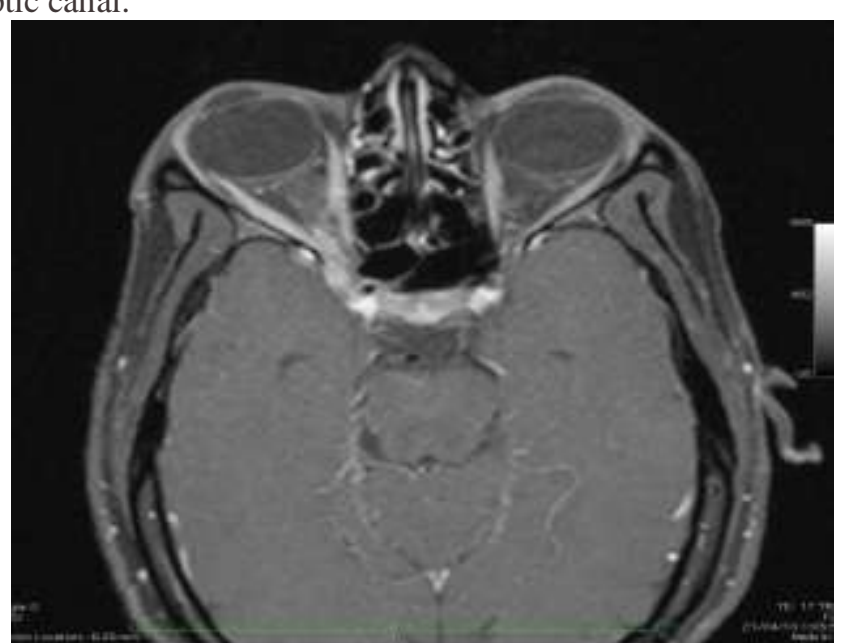

Fig 2b MRI Post contrast shows diffuse intense enhancement of the same lesion with moderate compression over the left optic nerve.

\section{Discussion}

Tolosa-Hunt syndrome (THS), a steroid-responsive painful ophthalmoplegia secondary to idiopatic granulomatous inflammation of the cavernous sinus or orbital apex( Kwan1988, Goadsby and Lance 1989), was first described by Tolosa ( 1954). Hunt et al (1961) reported six cases of painful ophthalmoplegia; that rapidly improved with the use of steroids. The criteria for THS was first described in the International Headache Society (IHS) in 1988 (Lance and Olesen ). This was later modified in the revised IHS headache classification of 2004 (Lance and Olesen, ). THS can be classified (La Mantia et al,2006) according to neuroimaging as benign (when no abnormal neuroimaging can be found), inflammatory (when inflammatory findings are shown on MRI or biopsy) and symptomatic (when neuroimaging reveals specific lesion). THS is basically a diagnosis of exclusion; diagnostic work-up (Gladstone 2007) includes routine blood work, inflammatory markers, fasting glucose, CSF evaluation, ANA, anti-dsDNA, c-ANCA, MRI, conventional angiography or MRA; and in some cases biopsy. Treatment should be with high dose steroids $(1 \mathrm{mg} / \mathrm{kg} / \mathrm{d})$ tapered slowly over 2 to 3 months .

Recurrence predominantly involving same eye is well described in the the criteria of THS. However compromise of the contralateral side, known as alternating THS is very rare and is not so frequently seen and examined using MRI. In our case impairment of different eyes at a prolonged interval of two years are seen clinically and by magnetic resonance imaging (Fig 1 and Fig 2) .Patient had involvement of one more ocular nerves on the different sides and lesions are well documented on the MRI .As the patient improved completely on steroids on both the occasions, we assume it to be a case of alternating THS. On review of published literature ,only two articles mentioned about MRI documented alternating THS out of the 233 articles of THS from 1966 to 2013. Lapresle (1975) reported a case of alternating THS diagnosed with carotid and vertebral angiography, orbital phlebography and air encephalography. Similarly Ohyama (1989) described a case of alternating THS diagnosed with cavernous sinus and orbital venography but the patient turned out to be diabetic. Few reports are mentioned in the literature as alternating THS (Ichikawa 1987 Freeman 1980) but the follow up revealed it as a sellar mass. Recently Guedes et al (2010) and Navi BB ( 2010) reported separately, MRI documented case reports of alternating THS. Both the authors argued theirs to be first reported MRI case of alternating THS. To the best our knowledge there is no other MRI documented case of alternating THS since then, hence we wish to highlight this case.

We suggest non-diabetic opthalmoplegia with documented MRI cavernous and orbital lesions which respond to steroids to be reported more often for the better understanding THS.

\section{References}

[1] Gladstone JP(2007). An Approach to the Patient With Painful Ophthalmoplegia, with a Focus on Tolosa-Hunt Syndrome.Curr Pain Headache Rep .11:317-25.

[2] Kwan ES, Wolpert SM, Hedges TR III, Laucella M (1988). Tolosa-Hunt syndrome revisited: not necessarily a diagnosis of exclusion . Am J Roentgenol .88(150):413-418.

[3] Goadsby PJ, Lance JW 1989. Clinicopathological correlation in a case of painful ophthalmoplegia: Tolosa-Hunt syndrome. J Neurol Neurosurg Psychiatry. 2:1290-1293.

[4] Tolosa E 1954. Periarteritic lesions of the carotid siphon with the clinical features of a carotid infraclinoid aneurysm. $J$ Neurol Neurosurg Psychiatry. 17:300-302. 
[5] Hunt WE, Meagher JN, LeFever HE, Zeman W(1961). Painful ophthalmoplegia.Its relation to indolent inflammation of the cavernous sinus. Neurology.11:56-62.

[6] Lance JW,Olesen J.(1988). Classification and diagnostic criteria for headache disorders, cranial neuralgias and facial pain. Headache Classification Committee of the International Headache Society. Cephalalgia.88(8):1-96.

[7] Lance JW, Olesen J(2004). The International Classification of Headache Disorders ICHD-II. Cephalalgia. $24: 131$.

[8] La Mantia L, Curone M, Rapoport AM, Bussone G: Tolosa-Hunt syndrome: critical literature review based on IHS 2004 criteria (2006). Cephalalgia. 26:772-781

[9] Ohyama M.Tezuka H,Michikawa K,Fukuchi T,Terashi A :Rinsho shinkeigaku = clinical neurology 29:3 1989 Mar pg 315-7

[10] Babak B Navi, Joseph E Safdieh.(2010). Recurrent ,alternating Tolosa-Hunt Syndrome. Neurologist.16(1):54-5.

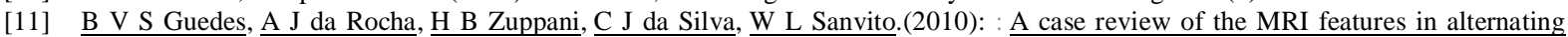
Tolosa-Hunt syndrome. Cephalalgia.30(9):1133-612. Lapresle J, Said G(1975) : Painful, alternating and recurrent ophthalmoplegia. Contribution to the study of Tolosa-Hunt syndrome.Rev Neurol (Paris).131(8):583-8.

[12] Freeman NR, Shraberg D(1980). Southern Medical Journal.73 (10):1398-1400

[13] Ichikawa N, Kohnosu T, Tuburaya K, Yoshimoto T(1987). Alternating Tolosa-Hunt syndrome with sellar destruction.. Rinsho Shinkeigaku.27(8):990-5. 